Letter to the Editor

\title{
Photodynamic diagnosis of metastatic lymph nodes using 5 -aminolevulinic acid in mouse squamous cell carcinoma
}

Jun Asai, M.D., Ph.D. ${ }^{a{ }^{*}}$, Yoshinori Harada, M.D., Ph.D. ${ }^{\text {b1 }}$, Masatomo Beika, M.D. ${ }^{b}$, Hideya Takenaka M.D., Ph.D. ${ }^{\text {a }}$, Norito Katoh M.D., Ph.D. ${ }^{\mathrm{a}}$, Tetsuro Takamatsu M.D., Ph.D. ${ }^{\mathrm{b}}$

${ }^{a}$ Department of Dermatology, Graduate School of Medical Science, Kyoto Prefectural University of Medicine, Kyoto, Japan

${ }^{\mathrm{b}}$ Department of Pathology and Cell Regulation, Graduate School of Medical Science, Kyoto Prefectural University of Medicine, Kyoto, Japan

\section{*Correspondence to:}

Jun Asai

Department of Dermatology, Graduate School of Medical Science, Kyoto Prefectural University of Medicine, 465 Hirokoji, Kawaramachi, Kamigyo-Ku, Kyoto, 602-8566, Japan

Tel \& Fax: +81-75-251-5586

E-mail: jasai@koto.kpu-m.ac.jp

${ }^{1}$ These authors equally contributed to this work.

Funding sources: This work was supported by grants from the Ministry of Education, Culture, Sports, Science and Technology of Japan (J.A., N.K.) and project of the Japan Science and Technology Agency (JST), Japan (J.A.)

Conflict of interest: The following authors declare conflicts of interest relevant to this publication: Yoshinori Harada: Ushio Incorporated; Tetsuro Takamatsu: Ushio Incorporated. The other authors declare no conflict of interest.

Text word count: 917 words

Number of references: 9

Number of figures: 2 
To the Editor,

Squamous cell carcinoma (SCCs) is one of the most frequent types of non-melanoma skin cancer. In certain cases, SCCs spread to the regional lymph nodes (LNs), which results in poor prognosis. It is essential to accurately evaluate $\mathrm{LN}$ metastasis in order to select the appropriate therapeutic strategy and predict the outcomes of the patients with SCCs. In addition to using sentinel lymph node biopsy (SLNB), which is a potentially promising procedure for assessing LN metastasis in patients with SCCs[1], achieving accurate and rapid intraoperative diagnosis is vital to ensure the use of a less invasive surgery.

The photosensitizer 5-aminolevulinic acid (5-ALA) has recently been clinically used for detecting the primary lesion in certain types of non-melanoma skin cancers-this process is termed as photodynamic diagnosis. The exogenous administration of 5-ALA causes selective accumulation of the heme precursor protoporphyrin IX (PpIX) in cancer cells.[2] PpIX is a fluorescent substance that emits a strong red fluorescence at approximately $635 \mathrm{~nm}$ on blue light excitation.[3, 4]

In the present study, we aimed to evaluate the feasibility of using 5-ALA-induced PpIX fluorescence as a rapid intraoperative diagnostic tool for LN metastasis in mouse SCCs.

A mouse model of SCCs with LN metastasis was established, as previously described by Matsumoto et al. [5] with minor modifications. In brief, NR-S1M cells $\left(6 \times 10^{6}\right.$ cells) were intracutaneously injected into the back area-adjacent to the tail—of 6-8-week-old C3H/He mice (SHIMIZU Laboratory Supplies, Kyoto, Japan). Three to 4 weeks after tumor implantation, the mice were administered 5-ALA hydrochloride (Wako Pure Chemical Industries, Kyoto, Japan) at a dose of $250 \mathrm{mg} / \mathrm{kg}$ body weight through a tail vein injection.[6] Six to 9 hours after the injection, the inguinal, axillary, and para-aortic LNs were excised and examined using fluorescence stereomicroscopy, as described previously.[6, 7] Briefly, light emitting from a mercury lamp was filtered through a $405 \pm 10 \mathrm{~nm}$ band-pass filter and used for excitation. The fluorescent emission at a wavelength longer than $430 \mathrm{~nm}$ was transmitted through a long-pass filter and detected by charge-coupled device camera. We performed intensity analysis by using Image-J software (National Institutes of Health, Bethesda, MD), as described previously.[7] The absolute mean R value and the signal intensity ratio of red to the sum of red, green, and blue $(R /[R+G+B])$ were used as indices 
corresponding to the red fluorescence of PpIX. All procedures were performed in accordance with the guidelines of the Animal Care and Use Committees of Kyoto Prefectural University of Medicine.

In total, 33 LNs obtained from 7 mice were examined, 12 of which were metastatic LNs. Strong accumulation of PpIX fluorescence was detected within the metastatic lesions in metastatic LNs (Figure 1A). Dimly weak to moderate fluorescence in the entire LN was detected in most of the non-metastatic inguinal or axillary LNs (Figure 1B). However, para-aortic LNs without metastasis showed no fluorescence or imperceptible fluorescence (Figure 1C). Compared to that in non-metastatic LNs, the $\mathrm{R}$ value (Figure $2 \mathrm{~A}$ ) and $\mathrm{R} /(\mathrm{R}+\mathrm{G}+\mathrm{B}$ ) ratio (Figure $2 \mathrm{~B}$ ) indicated significant increments in metastatic LNs (R value: metastatic LNs, $69.92 \pm 38.29$ vs. non-metastatic LNs, 23.73 $\pm 19.07, P<0.001 ; \mathrm{R} /(\mathrm{R}+\mathrm{G}+\mathrm{B})$ ratio: metastatic LNs, $0.782 \pm 0.153$ vs. non-metastatic LNs, 0.501 $\pm 0.154, P<0.001)$

Thus, we observed that the evaluation of 5-ALA-induced PpIX fluorescence can be a useful method for detecting metastatic LNs in a mouse SCC model. To our knowledge, this is the first report that applied photodynamic diagnosis using 5-ALA for the detection of LN metastasis in SCCs. Histological analysis is commonly performed for detecting LN metastasis through rapid intraoperative diagnosis. However, several studies have reported that rapid intraoperative pathological diagnosis is associated with a significant risk of overlooking metastasis, as only a few hematoxylin and eosin-stained slides from whole LNs are examined during routine histological examinations.[8, 9]

In addition to SCCs, photodynamic diagnosis or therapy of the primary lesions is widely used for non-melanoma skin cancers as well, including extramammary Paget disease, wherein LN metastasis would result in a poor prognosis. As fluorescence imaging methods are simple, rapid, and easily applicable, we believe that the 5-ALA-induced PpIX fluorescence method for rapid intraoperative diagnosis can be a novel and promising tool for patients with various types of non-melanoma skin cancers.

\section{Acknowledgements}

This work was supported in part by grants from the Ministry of Education, Culture, Sports, 
Science and Technology of Japan (J.A., N.K.) and project of the Japan Science and Technology Agency (JST), Japan (J.A.). We thank Mr. Shin-ichiro Kotani for assistance with fluorescence stereomicroscopy. 


\section{References}

[1] Matthey-Gie ML, Boubaker A, Letovanec I, Demartines N, Matter M: Sentinel lymph node biopsy in nonmelanoma skin cancer patients. J Skin Cancer 2013: 267474, 2013.

[2] Peng Q, Berg K, Moan J, Kongshaug M, Nesland JM: 5-Aminolevulinic acid-based photodynamic therapy: principles and experimental research. Photochem Photobiol 65: 235-251, 1997.

[3] Leunig A, Rick K, Stepp H, Gutmann R, Alwin G, Baumgartner R, et al.: Fluorescence imaging and spectroscopy of 5-aminolevulinic acid induced protoporphyrin IX for the detection of neoplastic lesions in the oral cavity. Am J Surg 172: 674-677, 1996.

[4] Harada K, Harada Y, Beika M, Koizumi N, Inoue K, Murayama Y, et al.: Detection of Lymph Node Metastases in Human Colorectal Cancer by Using 5-Aminolevulinic Acid-Induced Protoporphyrin IX Fluorescence with Spectral Unmixing. Int J Mol Sci 14: 23140-23152, 2013.

[5] Matsumoto G, Yajima N, Saito H, Nakagami H, Omi Y, Lee U, et al.: Cold shock domain protein A (CSDA) overexpression inhibits tumor growth and lymph node metastasis in a mouse model of squamous cell carcinoma. Clin Exp Metastasis 27: 539-547, 2010.

[6] Murayama Y, Harada Y, Imaizumi K, Dai P, Nakano K, Okamoto K, et al.: Precise detection of lymph node metastases in mouse rectal cancer by using 5-aminolevulinic acid. Int J Cancer 125: 2256-2263, 2009.

[7] Koizumi N, Harada Y, Murayama Y, Harada K, Beika M, Yamaoka Y, et al.: Detection of 
metastatic lymph nodes using 5-aminolevulinic acid in patients with gastric cancer. Ann Surg Oncol 20: 3541-3548, 2013.

[8] Ghossein RA, Rosai J: Polymerase chain reaction in the detection of micrometastases and circulating tumor cells. Cancer 78: 10-16, 1996.

[9] Natsugoe S, Aikou T, Shimada M, Yoshinaka H, Takao S, Shimazu H, et al.: Occult lymph node metastasis in gastric cancer with submucosal invasion. Surg Today 24: 870-875, 1994. 


\section{Figure Legends}

Figure 1. Representative images of metastatic lymph nodes (A) and non-metastatic inguinal (B) and para-aortic (C) lymph nodes. White-light and fluorescence images (left panels, white images; middle panels, fluorescence images, scale bar: 1mm) and hematoxylin and eosin-stained sections (right panels, original magnification: $\times 12.5$ ) of each lymph node are shown. The yellow arrows indicate metastatic foci. Red fluorescence, representative of metastatic foci, is observed in the metastatic lymph node (A). Microscopic fluorescence images: excitation, 395-415 nm; emission, >430 nm.

Figure 2. The absolute mean $\mathrm{R}$ value $(\mathrm{A})$ and the signal intensity ratio of red to the sum of red, green, and blue $(\mathrm{R} /[\mathrm{R}+\mathrm{G}+\mathrm{B}])$ (B) between metastatic lesions of metastatic lymph nodes and non-metastatic lymph nodes. $(*, P<0.001)$ 

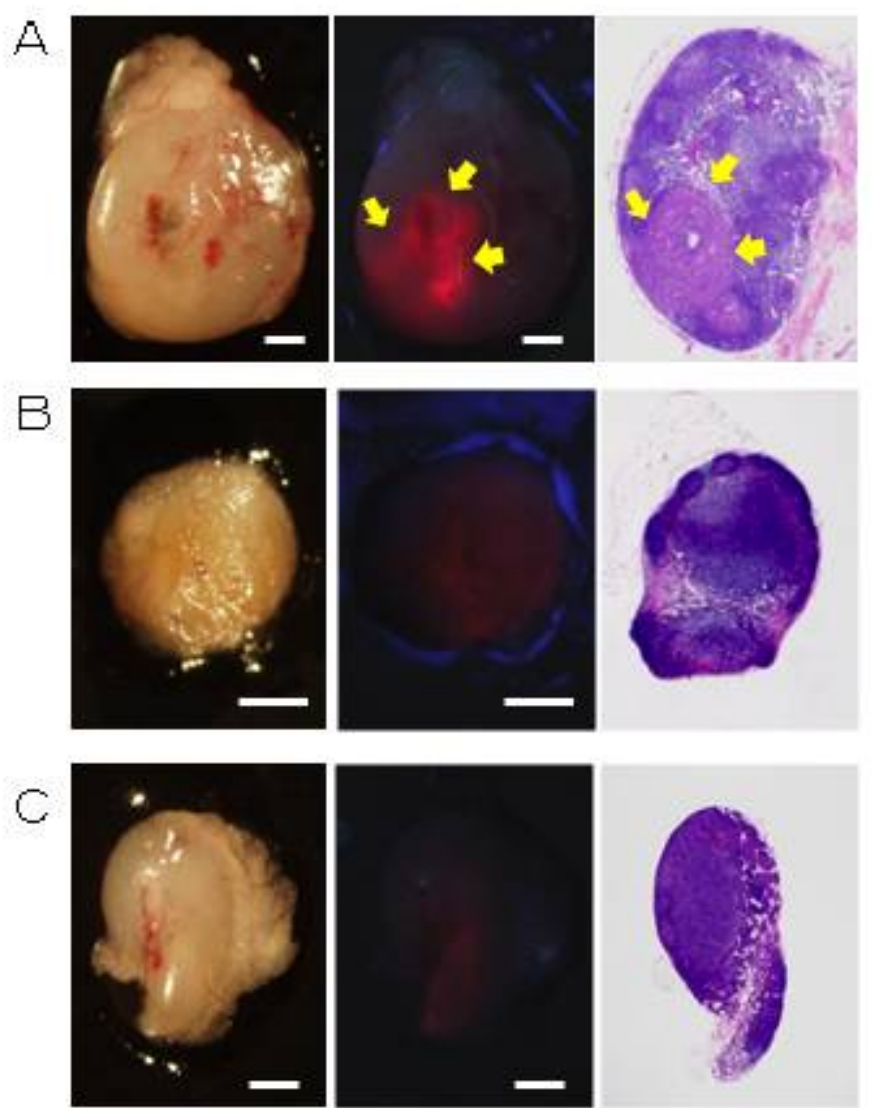

Figure 1 
A

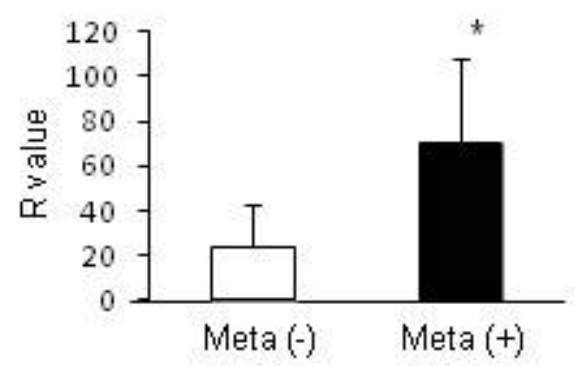

B

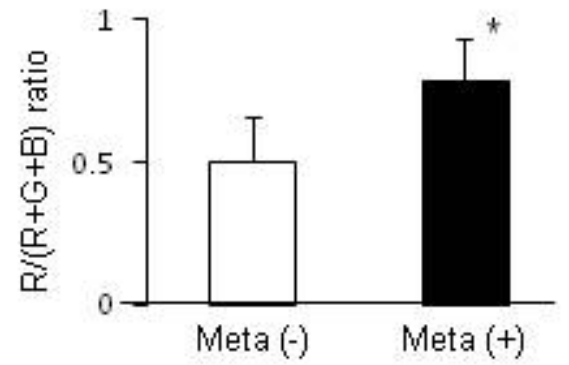

Figure 2 